\title{
Is There an Incremental Prognostic Value of Evaluating Left Ventricular Dyssynchrony by Gated SPECT in Patients with Systolic Heart Failure and Altered Myocardial Sympathetic Innervation as Evaluated by Cardiac I-123 mIBG Imaging?
}

\author{
Akif Mohammed, Gordon Jacobsen, Karthik Ananthasubramaniam* \\ Heart and Vascular Institute and the Department of Biostatistics and Epidemiology, Henry Ford Hospital, \\ Detroit, MI, USA \\ Email: *kananth1@hfhs.org
}

Received 18 January 2016; accepted 17 July 2016; published 20 July 2016

Copyright (C) 2016 by authors and Scientific Research Publishing Inc.

This work is licensed under the Creative Commons Attribution International License (CC BY).

http://creativecommons.org/licenses/by/4.0/

c) (i) Open Access

\section{Abstract}

Background: Altered myocardial sympathetic innervation activity (AMSI) is known to be present in systolic heart failure patients (SHF) and recently SPECT imaging using I-123 mIBG heart to mediastinum $(\mathrm{H} / \mathrm{M})$ ratio $<1.6$ has been shown to predict MACE in the ADMIRE-HF trial. Left ventricular mechanical dyssynchrony (LVMD) is known to be present in a substantial number of SHF patients and has been studied mainly to guide CRT therapy. Recently gated SPECT has shown promise to provide an accurate assessment of LVMD. It remains unclear how the combination of AMSI and LVMD collectively affect clinical outcomes and other cardiovascular parameters. Objectives: The objectives are to examine the clinical characteristics and incremental prognostic value for MACE of LVMD determined by SPECT in SHF patients with or without abnormal cardiac MIBG uptake $(\mathrm{H} / \mathrm{M}$ ratio < 1.6). Methods: Out of $30 \mathrm{SHF}$ patients who participated from our institution in the ADMIRE-HF trial studying MIBG based AMSI, we included 22 patients with abnormal MIBG H/M ratio of $<1.6$. We performed gated SPECT LVMD analysis on these patients using the Emory Cardiac Toolbox. The 2 SPECT variables for LVMD assessed were histogram bandwidth and phase standard deviation both of which assess the extent of dispersion of $L V$ activation during contraction as a marker of LVMD. Patients were followed up for a mean period of 6 years. The primary end point was mortality from any cause and secondary end point was heart failure admission or myocardial

\footnotetext{
*Corresponding author.

How to cite this paper: Mohammed, A., Jacobsen, G. and Ananthasubramaniam, K. (2016) Is There an Incremental Prognostic Value of Evaluating Left Ventricular Dyssynchrony by Gated SPECT in Patients with Systolic Heart Failure and Altered Myocardial Sympathetic Innervation as Evaluated by Cardiac I-123 mIBG Imaging? World Journal of Nuclear Science and Technology, 6, 161-169. http://dx.doi.org/10.4236/wjnst.2016.63018
} 
infarction or ICD shock. Results: 2 Groups were defined: Group A: $n=17$ with H/M MIBG ratio $<1.6$ and +LVMD and Group B, $n=5$ H/M MIBG ratio < 1.6 and -LVMD. Baseline characteristics, cardiac risk factors and medications were comparable between both groups. LVEF was lower and RBBB was less common in Group A. There was no statistical difference in achievement of primary or secondary end points in the two groups including death heart failure readmissions, ICD shocks or MI. Conclusions: In our pilot study, we did not find definitive value of adding SPECT based LVMD to abnormal cardiac MIBG imaging in SHF patients with regards to predicting outcomes. Although our sample size is too small to make any definitive conclusions, it is possible that LVMD works independently through different pathways in the progression of SHF and hence may not necessarily add incremental value to AMSI determination using MIBG.

\title{
Keywords
}

\author{
SPECT Dyssynchrony, I-123 mIBG, Myocardial Sympathetic Innervation, Heart to Mediastinum \\ Ratio
}

\section{Introduction}

Compared with myocardium of healthy controls, the myocardium of patients with chronic left ventricular dysfunction is characterized by a significant reduction of pre-synaptic norepinephrine (NE) uptake and post-synaptic beta-adrenoceptor density [1] [2]. The uptake-1 mechanism is one of the main NE disposal systems, and its malfunction in chronic systolic heart failure (SHF) may lead to abnormal catecholamine concentration in the synaptic cleft and reduced concentration in the pre synaptic adrenergic nerve endings [3]. I-123 mIBG was developed through a modification of the potent neuron-blocking agent guanethidine that acts selectively on sympathetic nerve endings. Uptake of I-123 mIBG into neurons is achieved mainly through the uptake- 1 mechanism, a homeostatic system responsible for the reuptake of NE. Unlike NE, I-123 mIBG is not metabolized, allowing it to be imaged to study altered myocardial sympathetic innervations activity (AMSI). I-123 mIBG uptake is semi-quantified by calculating a heart-to-mediastinum ratio (HMR) after drawing regions of interest over the heart and mediastinum (Figure 1). This approach provides a highly reproducible index of cardiac sympathetic activity [4]. By comparing early and delayed activities, the I-123 mIBG washout (WO) rate from the myocardium can be derived, providing a parameter that reflects retention of NE by sympathetic neurons. It has been shown that the uptake of I-123-mIBG is significantly reduced in patients with SHF. Impaired cardiac adrenergic innervation as assessed by ${ }^{123} \mathrm{I}$-mIBG imaging is strongly related to mortality in patients with HF independently of its cause, and I-123 mIBG heart to mediastinum ratio $<1.6$ has been shown to predict MACE in the ADMIRE-HF trial [5]. A pictorial illustration of $\mathrm{H} / \mathrm{M}$ ratio measurement and calculation is shown in Figure 1.

\section{SPECT Dyssynchrony}

Assessment of LV mechanical dyssynchrony (LVMD) can be done using phase analysis of gated SPECT MPI. Phase analysis is based on the partial volume effect, which states that LV regional maximal counts in SPECT MPI images are nearly proportional to the myocardial wall thickness of the same region [6]. Phase standard deviation (the standard deviation of the OMC phase distribution) (PSD) and phase histogram bandwidth (the width of the histogram band, (PHB) are two quantitative indices to assess LV global mechanical dyssynchrony. Normal limits for these indices have been generated from prior gated SPECT MPI studies [7] [8].

Processing steps of phase analysis of gated SPECT MPI uses Emory Cardiac Tool Box (ECTB), Emory University/Syntermed, Inc., Atlanta, GA. The gated SPECT MPI data are reconstructed and reoriented to generate a gated short-axis image. 3D sampling is performed on each temporal frame of the gated short-axis image to detect regional maximum counts. The points shown in the plots are regional wall thickening data. The first harmonic Fourier function is used to approximate the wall thickening data (shown as the solid line) to calculate a phase angle for each region. Once the phase angles of all regions are obtained, a phase distribution is generated and displayed in polar map or in histogram. Note that the phase difference between 8 and 16 frame/cycle is very 


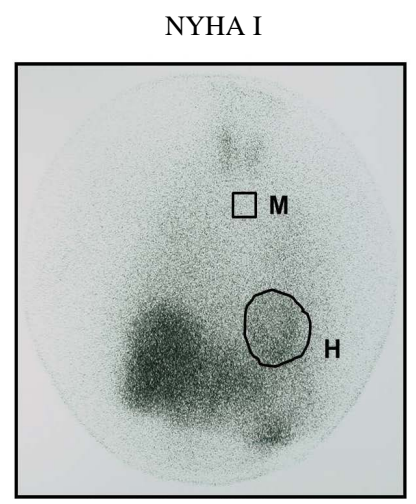

H/M: 2.34, WR: $15 \%$

(a)
NYHA III

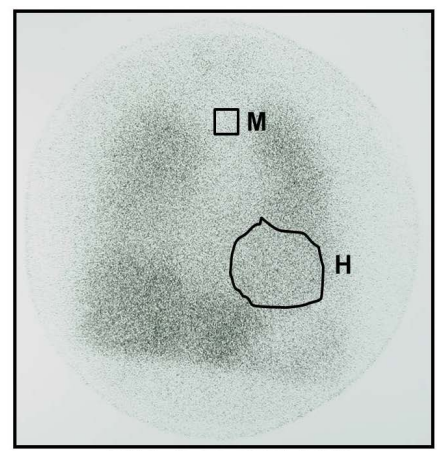

H/M: 1.45, WR: 39\%

(b)

Washout Rate $=\frac{\operatorname{Early}(\mathrm{H}-\mathrm{M})-\text { Delayed }(\mathrm{H}-\mathrm{M})}{\operatorname{Early}(\mathrm{H}-\mathrm{M})}$

H: heart; M: mediastinum; H/M: heart to mediastinum activity ratio; ${ }^{123}$ I-MIBG: Iodine-123 Metaiodobenzylguanidine; NYHA: New York Heart Association.

Figure 1. ${ }^{123}$ I-MIBG imaging. (a) A case of NYHA functional class I status. (b) A case of NYHA functional class III status (Reproduced with permission from [17].

small $-0.5^{\circ}\left(360^{\circ}\right.$ corresponding to one cardiac cycle) demonstrating that the first harmonic approximation improves the temporal resolution of the phase measurement. Also note that the phase polar map shows a significant phase delay (bright region) at the anterior and apical wall, where the perfusion polar map shows a severe defect.

The developed OMC normal databases consisted of phase SD $\left(14.2^{\circ} \pm 5.1^{\circ}\right.$ for men and $11.8^{\circ} \pm 5.2^{\circ}$ for women), and phase histogram bandwidth $\left(38.7^{\circ} \pm 11.8^{\circ}\right.$ for men and $30.6^{\circ} \pm 9.6^{\circ}$ for women) [9].

The relationship between cardiac sympathetic innervation and myocardial dyssynchrony is unclear and whether presence of LVMD has additional prognostic value for outcomes has not been well documented and formed the basis of out retrospective single center analysis.

\section{Methods}

This was a retrospective cohort study. Out of 43 SHF patients who participated in the ADMIRE-HF trial, 30 patients with gated SPECT data were initially included in the study. Out of 30 we included 22 patients with abnormal I-123 mIBG H/M ratio of $<1.6$ studying the final study group. We then performed gated SPECT LVMD analysis on these patients using the ECTB software. Each subject enrolled in this study underwent a standard resting ECG-gated SPECT myocardial perfusion scan per standard American Society of Nuclear Cardiology guidelines for SPECT acquisition as part of the ADMIRE-HF trial [10].

Gated SPECT data were acquired at 8 frames per cardiac cycle. The R-R window used for gating was averaged from the prescan electrocardiogram. The short-axis data sets were generated by Butterworth filtering, followed by filtered back projection reconstruction and oblique reorientation. The ECTB software then extracts 3-dimensional count distributions from each of the 8 left ventricular short axis data sets and submits for Fourier phase analysis. Then, the analysis generates a 3-dimensional phase distribution that describes the timing of the left ventricular regional $\mathrm{OMC}$ as a function of degrees, with the $360^{\circ}$ range representing the entire length of the $\mathrm{R}-\mathrm{R}$ interval. Once the phase distribution was generated, it is displayed on the polar map as well as in histogram format (Figure 2).

The $\mathrm{x}$-axis represents the timing of 1 cardiac cycle (R-R interval) in degrees. The y-axis represents the percent of myocardium that demonstrated the OMC during any particular time of the cardiac cycle. The 2 SPECT variables for LVMD assessed were histogram bandwidth and phase standard deviation both of which assess the extent of dispersion of LV activation during contraction as a marker of LVMD. The software has been implemented 

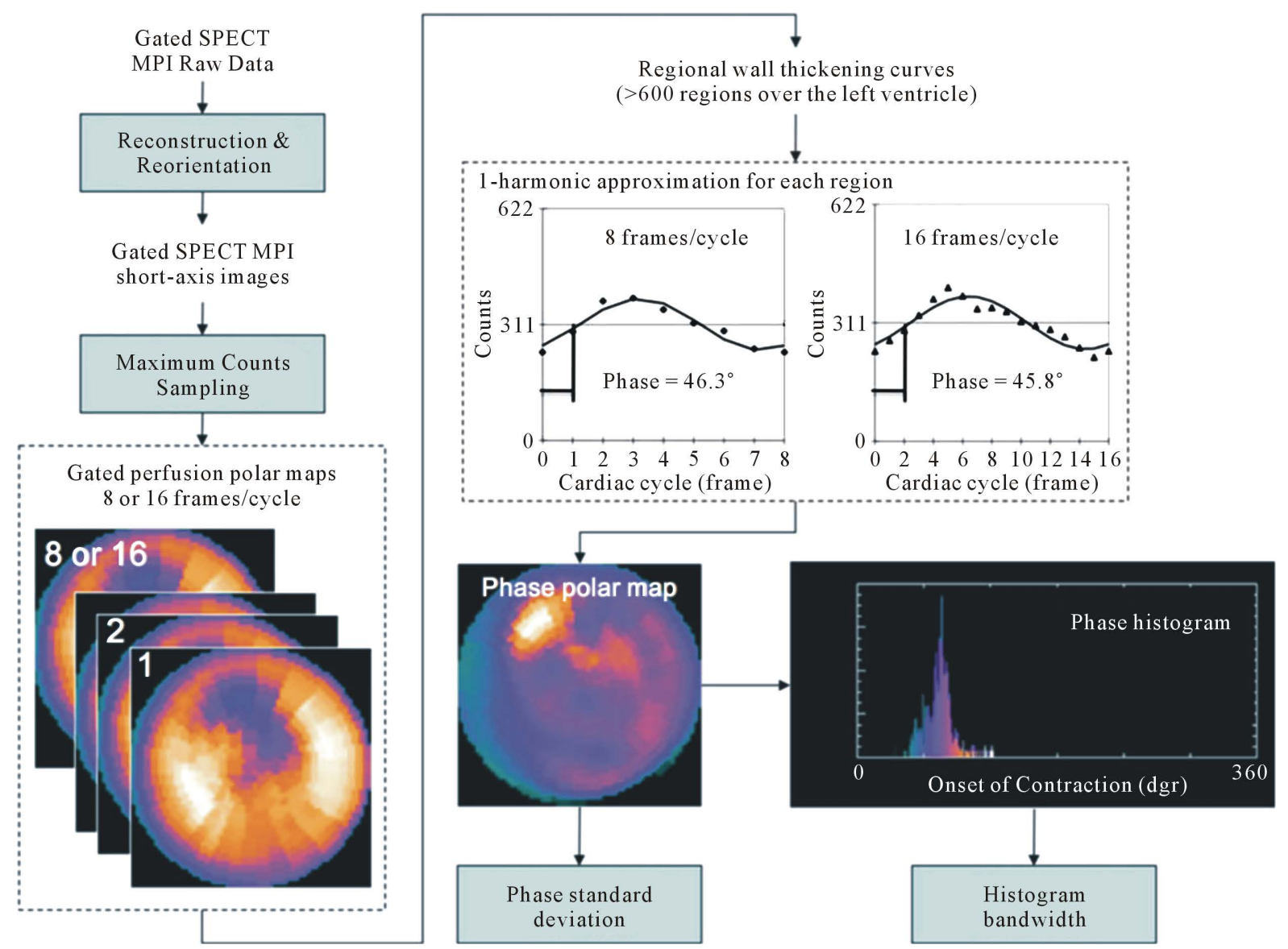

Figure 2. SPECT dyssynchrony methodology (Reproduced with permission from [18]).

in the Emory Cardiac Toolbox (Emory University/Syntermed, Inc., Atlanta, GA) for analysis of gated SPECT myocardial perfusion studies.

The primary end point was mortality from any cause and secondary end point was heart failure admission or myocardial infarction or ICD shock. Myocardial infarction was defined as detection of a rise and/or fall of the measurements of specific biomarker cardiac troponin in the clinical setting of acute myocardial ischemia. Admission for decompensated heart failure was defined as admission with dyspnea requiring administration of intravenous diuretics. ICD shock was defined as appropriate therapy received by patient for ventricular tachycardia or ventricular fibrillation as documented on interrogation of the ICD. Patients were followed up for a mean period of 6 years.

\section{Statistical Analysis}

The Fisher exact test has been used for comparisons of all of the categorical variables, the Cochran-Armitage trend test has been used for comparisons of ordinal variables, two-sample t-tests have been used for comparisons of numeric variables containing normally distributed data, and the Wilcoxon rank test has been used for comparisons of numeric variables containing non-normally distributed data. Differences in outcomes were evaluated between the 2 groups using Fisher exact test.

\section{Results}

The baseline clinical and echocardiographic parameters are summarized in Table 1. 2 Groups were defined: (Group A: $\mathrm{n}=17$ with H/M I-123 mIBG ratio <1.6 and +LVMD and Group B: $\mathrm{n}=5, \mathrm{H} / \mathrm{M}$ MIBG ratio <1.6 and -LVMD). Baseline characteristics, cardiac risk factors and medications were comparable between both groups. LVEF was lower and RBBB was less common in Group A. 
Table 1. Baseline and echocardiographic characteristics of patients with and without SPECT Dyssynchrony.

\begin{tabular}{|c|c|c|c|c|}
\hline Variable & Response & $\begin{array}{c}\text { H/M Ratio < } 1.6 \text { without } \\
\text { SPECT Dyssynchrony } \\
(\mathrm{N}=5)\end{array}$ & $\begin{array}{l}\text { H/M Ratio }<1.6 \text { with } \\
\text { SPECT Dyssynchrony } \\
\quad(\mathrm{N}=17)\end{array}$ & $\begin{array}{c}\text { Comparison } \\
\text { p-value }\end{array}$ \\
\hline Male Gender & Yes & $4(80.0 \%)$ & 11 (64.7\%) & $0.637(\mathrm{~F})$ \\
\hline Black Race & Yes & $5(100.0 \%)$ & $16(94.1 \%)$ & $1.000(\mathrm{~F})$ \\
\hline \multirow{3}{*}{ Smoking Status } & Never Smoked & $1(20.0 \%)$ & $3(17.6 \%)$ & \multirow{3}{*}{0.728 (C-A) } \\
\hline & Ex-Smoker & $2(40.0 \%)$ & 10 (58.8\%) & \\
\hline & Current Smoker & $2(40.0 \%)$ & $4(23.5 \%)$ & \\
\hline DIABF & Yes & $1(20.0 \%)$ & $6(35.3 \%)$ & $1.000(\mathrm{~F})$ \\
\hline DYSLIP & Yes & $5(100.0 \%)$ & 11 (64.7\%) & $0.266(\mathrm{~F})$ \\
\hline HTN & Yes & $5(100.0 \%)$ & 15 (88.2\%) & $1.000(\mathrm{~F})$ \\
\hline CKD & Yes & $3(60.0 \%)$ & $7(41.2 \%)$ & $0.624(\mathrm{~F})$ \\
\hline AFIB & Yes & $1(20.0 \%)$ & $3(17.6 \%)$ & $1.000(\mathrm{~F})$ \\
\hline COPD & Yes & $2(40.0 \%)$ & $4(23.5 \%)$ & $0.585(\mathrm{~F})$ \\
\hline LBBB & Yes & $0(0.0 \%)$ & $7(41.2 \%)$ & $0.135(\mathrm{~F})$ \\
\hline RBBB & Yes & $2(40.0 \%)$ & $0(0.0 \%)$ & $0.043(\mathrm{~F})^{*}$ \\
\hline \multirow{2}{*}{ NYHA } & II & $3(60.0 \%)$ & 15 (88.2\%) & \multirow{2}{*}{0.210 (C-A) } \\
\hline & III & $2(40.0 \%)$ & $2(11.8 \%)$ & \\
\hline \multirow{3}{*}{ NYHA Max } & II & $2(40.0 \%)$ & $6(37.5 \%)$ & \multirow{3}{*}{0.716 (C-A) } \\
\hline & III & $3(60.0 \%)$ & $8(50.0 \%)$ & \\
\hline & IV & $0(0.0 \%)$ & 2 (12.5\%) & \\
\hline Ischemic CHF & Yes & $2(40.0 \%)$ & 4 (23.5\%) & $0.585(\mathrm{~F})$ \\
\hline MI Hx & Yes & $1(20.0 \%)$ & $5(29.4 \%)$ & $1.000(\mathrm{~F})$ \\
\hline ICDENRF & Yes & $1(20.0 \%)$ & $4(23.5 \%)$ & $1.000(\mathrm{~F})$ \\
\hline AICD & Yes & $2(40.0 \%)$ & $8(47.1 \%)$ & $1.000(\mathrm{~F})$ \\
\hline BIV & Yes & $0(0.0 \%)$ & $3(17.6 \%)$ & $1.000(\mathrm{~F})$ \\
\hline ASA & Yes & $4(80.0 \%)$ & 14 (82.4\%) & $1.000(\mathrm{~F})$ \\
\hline Plavix & Yes & $1(20.0 \%)$ & $1(5.9 \%)$ & $0.411(\mathrm{~F})$ \\
\hline Ace-I & Yes & $5(100.0 \%)$ & 11 (64.7\%) & $0.266(\mathrm{~F})$ \\
\hline ARB & Yes & $0(0.0 \%)$ & $6(35.3 \%)$ & $0.266(\mathrm{~F})$ \\
\hline B-Blocker & Yes & $5(100.0 \%)$ & $16(94.1 \%)$ & $1.000(\mathrm{~F})$ \\
\hline Hydralazine Nitrate & Yes & $1(20.0 \%)$ & $7(41.2 \%)$ & $0.613(\mathrm{~F})$ \\
\hline Spironolactone & Yes & $2(40.0 \%)$ & $9(52.9 \%)$ & $1.000(\mathrm{~F})$ \\
\hline Digoxin & Yes & $0(0.0 \%)$ & $4(23.5 \%)$ & $0.535(\mathrm{~F})$ \\
\hline Statin & Yes & $3(60.0 \%)$ & 12 (70.6\%) & $1.000(\mathrm{~F})$ \\
\hline
\end{tabular}




\begin{tabular}{|c|c|c|c|c|}
\hline \multicolumn{5}{|l|}{ Continued } \\
\hline Lasix & Yes & $3(60.0 \%)$ & 13 (76.5\%) & $0.585(\mathrm{~F})$ \\
\hline HCTZ & Yes & $1(20.0 \%)$ & $1(5.9 \%)$ & $0.411(\mathrm{~F})$ \\
\hline Coumadin & Yes & $1(20.0 \%)$ & $1(5.9 \%)$ & $0.411(\mathrm{~F})$ \\
\hline Amiodarone & Yes & $2(40.0 \%)$ & $0(0.0 \%)$ & $0.043(\mathrm{~F})^{*}$ \\
\hline Contrast Agent & Yes & $0(0.0 \%)$ & 3 (18.8\%) & $0.549(\mathrm{~F})$ \\
\hline \multicolumn{2}{|c|}{ Age } & $63.2 \pm 14.6$ & $63.3 \pm 13.1$ & $0.989(\mathrm{~T})$ \\
\hline \multicolumn{2}{|c|}{ BMI } & $34.6 \pm 9.9$ & $31.1 \pm 8.8$ & $0.450(\mathrm{~T})$ \\
\hline \multicolumn{2}{|c|}{ LVEF } & $32.0 \pm 2.7$ & $21.5 \pm 6.4$ & $0.002(\mathrm{~T})^{*}$ \\
\hline \multicolumn{2}{|c|}{ VS SBP } & $112.0 \pm 13.5$ & $119.2 \pm 16.7$ & $0.387(\mathrm{~T})$ \\
\hline \multicolumn{2}{|c|}{ VS DBP } & $71.4 \pm 5.8$ & $71.9 \pm 11.5$ & $0.930(\mathrm{~T})$ \\
\hline \multicolumn{2}{|c|}{ VS Resp } & $20.6 \pm 5.4$ & $18.4 \pm 1.5$ & $0.597(\mathrm{~W})$ \\
\hline \multicolumn{2}{|c|}{ VSO2 Sat } & $92.6 \pm 5.0$ & $95.3 \pm 2.7$ & $0.125(\mathrm{~T})$ \\
\hline \multicolumn{2}{|c|}{ BSA } & $2.3 \pm 0.4$ & $2.0 \pm 0.4$ & $0.232(\mathrm{~T})$ \\
\hline \multicolumn{2}{|c|}{ Echo LV Mass } & $162.6 \pm 26.5$ & $207.0 \pm 33.4$ & $0.015(\mathrm{~T})^{*}$ \\
\hline \multicolumn{2}{|c|}{ Echo LVM BSA } & $73.6 \pm 16.1$ & $105.2 \pm 23.3$ & $0.012(\mathrm{~T})^{*}$ \\
\hline \multicolumn{2}{|c|}{ Echo LV EDV } & $164.5 \pm 43.2$ & $203.8 \pm 50.2$ & $0.136(\mathrm{~T})$ \\
\hline \multicolumn{2}{|c|}{ Echo LVEF } & $43.0 \pm 7.3$ & $34.0 \pm 4.7$ & $0.005(\mathrm{~T})^{*}$ \\
\hline \multicolumn{2}{|c|}{ MPI LVEF } & $38.0 \pm 6.3$ & $25.1 \pm 7.1$ & $0.002(\mathrm{~T})^{*}$ \\
\hline \multicolumn{2}{|c|}{ LVEF Follow-up minus Baseline } & $-1.0 \pm 13.2$ & $9.1 \pm 15.3$ & $0.287(\mathrm{~T})$ \\
\hline \multicolumn{2}{|c|}{ EDV Follow-up minus Baseline } & $-0.1 \pm 1.6$ & $-0.5 \pm 0.9$ & $0.566(\mathrm{~T})$ \\
\hline \multicolumn{2}{|c|}{ ESV Follow-up minus Baseline } & $0.8 \pm 2.2$ & $-0.5 \pm 0.9$ & $0.216(\mathrm{~T})$ \\
\hline
\end{tabular}

Categorical data is given as Frequency (Percent of Group). Numeric data is given as Mean \pm Standard Deviation. $(\mathrm{F})=$ Fisher Exact Test; $(\mathrm{C}-\mathrm{A})=$ Cochran-Armitage Trend Test; $(\mathrm{T})$ = Two-Sample T-Test; $(\mathrm{W})$ = Wilcoxon Rank Sum Test; * Statistically Significant, $\mathrm{p}<0.05$.

Within the total of 22 patients who had histogram bandwidth and standard deviation data along with an H/M ratio less than 1.6, the histogram bandwidth had a mean of $140.6 \pm 82.2$ while the phase standard deviation had a mean of $45.3 \pm 21.6$.

In the 2 groups studied, Group A had a mean histogram bandwidth of $164.8 \pm 78$ compared to a mean histogram bandwidth of $58.2 \pm 10.9$ for the 5 patients without LVMD ( $<0.001)$. In Group A the mean phase standard deviation was $52.8 \pm 18.6$ for the 17 patients with LVMD compared to $19.8 \pm 4.8$ for the 5 patients without abnormal SPECT $(\mathrm{p}<0.001)$.

A total of 12 patients had the combination outcome of death and/or ICD shock. Nine of them had death without ICD shock, 2 of them had ICD shock without death, and 1 of them had both death and ICD shock. Death/ ICD shock occurred in 2 of the 5 patients who had an H/M ratio less than 1.6 without SPECT dyssynchrony (40.0\%) and in 10 of the 17 patients who had an H/M ratio less than 1.6 with SPECT dyssynchrony (58.8\%). That difference is not statistically significant (Fisher exact test p-value $=0.624$ ). Scatter plots of events in relation to H/M ratio are shown in Figure 3(a) and Figure 3(b).

Regarding the composite secondary end point of MI/heart failure admission/ICD shock, 10/17 patients with abnormal SPECT had an event (58.8\%) compared to 3/5 patients without abnormal SPECT (60) but again this was not statistically significant $(\mathrm{p}$-value $=1.000)$. 


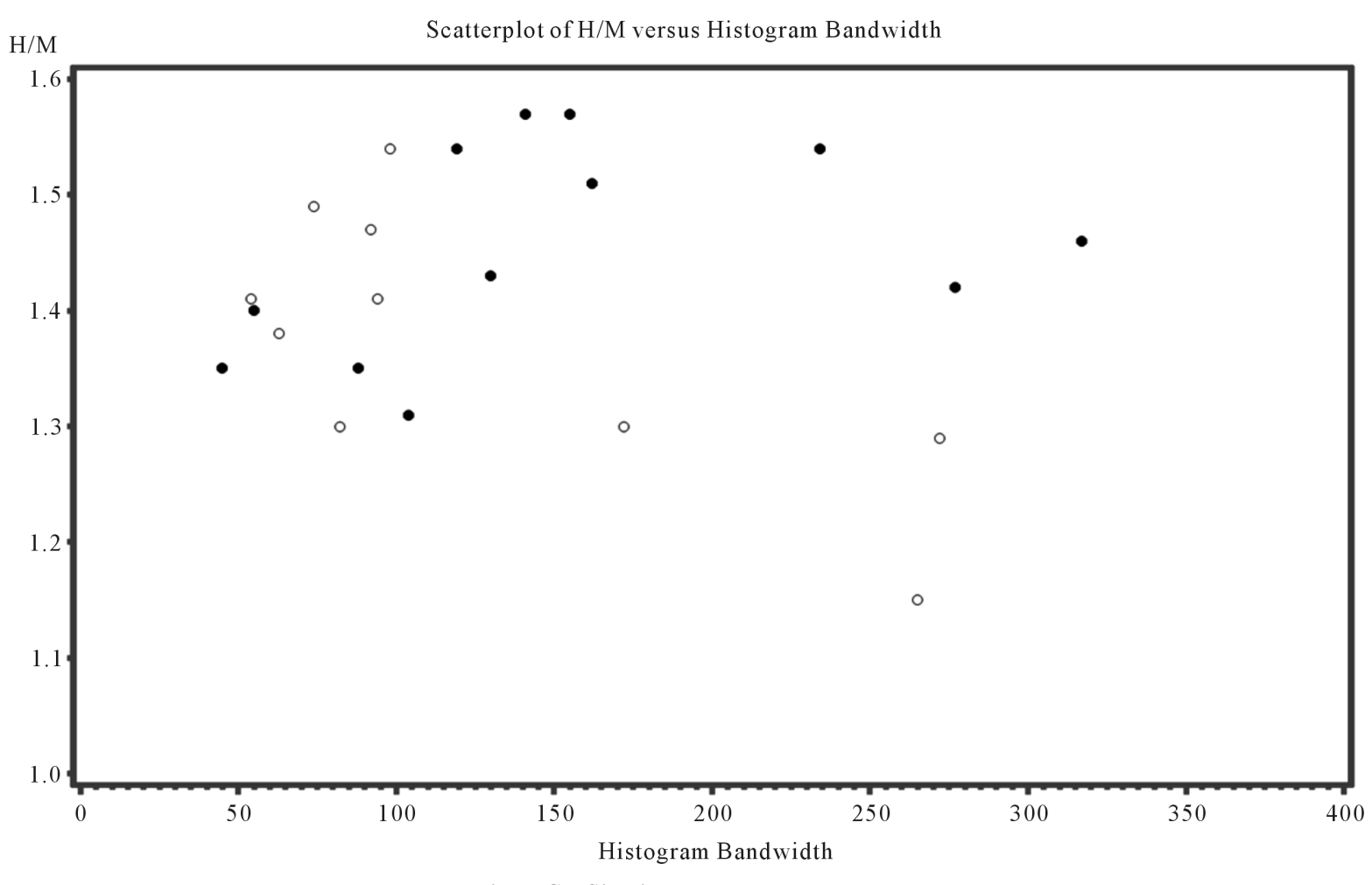

Death or ICD Shock $\circ \circ \circ$ No

• Yes

(a)

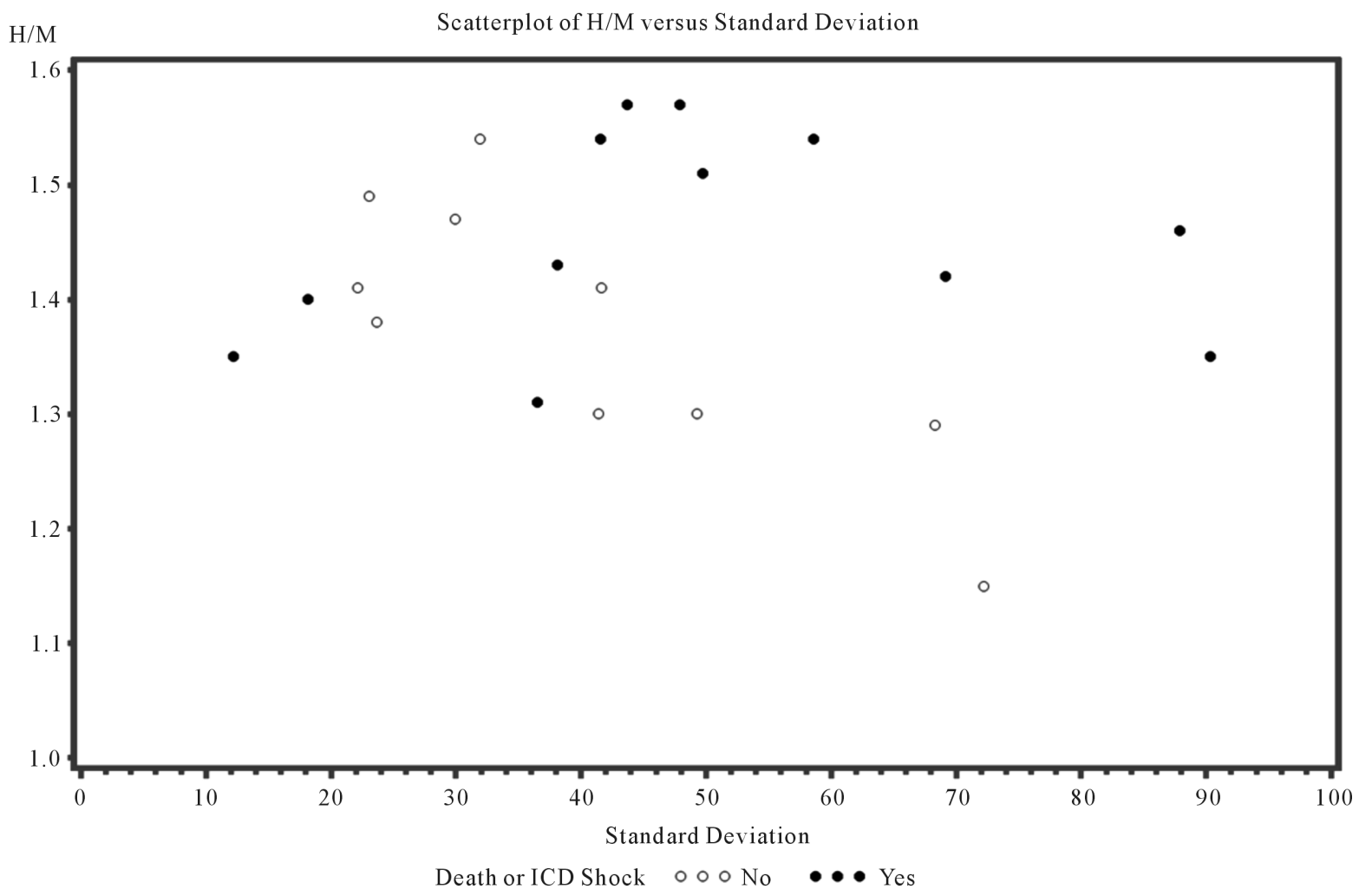

(b)

Figure 3. Scatter plots of cardiac events in relation to H/M ratio, histogram bandwidth (a) and phase standard deviation (b). 


\section{Discussion}

Impaired cardiac adrenergic innervation as assessed by I-123 mIBG imaging is strongly related to mortality in patients with HF independently of its cause from prior studies [5]. Use of I-123 mIBG is now an FDA approved indication for use in NYHA class 2 - 3 SHF patients with EF of 30\% - 35\% when additional risk stratification is desired prior to ICD placement. Patients with SHF have a higher likelihood of LVMD which is linked to sudden cardiac death. CRT is approved for the treatment of patients with NYHA class III/IV heart failure symptoms who have ejection fractions of 35\% or less and a QRS duration of greater than 120 milliseconds on a surface electrocardiogram. Several studies have shown benefits with CRT when added to optimal medical therapy for groups of patients who meet these selection criteria. These benefits include improved functional status, exercise tolerance, quality of life, and left ventricular reverse remodeling [11]-[14]. Prior studies have evaluated the relationship between LVMD and cardiac sympathetic innervation. In one study looking at relationship between MRI derived dyssynchrony parameters (spatial and temporal) and H/M ratio by I-123 mIBG in patients with nonischemic heart failure [15]. Spatial dyssynchrony was worse in patients with H/M ratio $<2$ but no differences in temporal dyssynchrony existed. Specific cutoffs of I-123 mIBG as used in our study were not evaluated. In another study looking at echocardiographic dyssynchrony and H/M ratio by I-123 mIBG [16] Overall dyssynchrony was present more in patients with lower compared to higher $\mathrm{H} / \mathrm{M}$ ratios. Interestingly those with dyssynchrony and $\mathrm{H} / \mathrm{M}$ ratio $>1.6$ had better CRT response than those without dyssynchrony but with $\mathrm{H} / \mathrm{M}$ ratio $<$ 1.6 [16]. An important substudy done in the ADMIRE HF trial was a dysynchrony analysis looking at 92 sudden cardiac death events in the primary trial over a period of 17 month of follow-up. A propensity matched control group of 85 patients were compared to these 92 patients. Compared to the control the phase standard deviation parameter of SPECT dyssynchrony was greater in those who experienced events and moreover degree of SPECT dyssynchrony was greater in those with $\mathrm{H} / \mathrm{M}$ ratio $<1.6$. However of note this study was done in a very sub selected and highest risk group of patients where hard events had occurred and did not address the role of SPECT dyssynchrony in all patients with low H/M ratio of $<1.6$. In our study we specifically looked at the abnormal H/M ratio < 1.6 and studied extent of SPECT dysynchrony and showed that the histogram bandwidth and the phase standard deviation are indeed more abnormal in the majority (77\%) of patients with $\mathrm{H} / \mathrm{M}$ ratio < 1.6 but overall not difference in events were detectable in both primary and secondary end points Although our sample size is too small to make any definitive conclusions it is possible that LVMD works independently through different pathways in the progression of SHF and hence may not necessarily add incremental value to AMSI determination using MIBG.

\section{Limitations}

Our study is single center with a small sample size limiting any definitive conclusions. Yet it is among the few focused studies which has evaluated the link between gated SPECT LVMD and AMSI and showed that indeed there is greater dyssynchrony as detected by SPECT in patients with AMSI as shown in prior studies but not necessarily predictive of events which could be related to sample size limitations.

\section{Conclusion}

Gated SPECT LVMD is prevalent in patients with SHF who have AMSI (H/M ratio with I-123 mIBG of <1.6). In our pilot study, we did not find definitive value of adding SPECT based LVMD to abnormal cardiac MIBG imaging in SHF patients with regards to predicting outcomes. Although our sample size is too small to make any definitive conclusions, it is possible that LVMD works independently through different pathways in the progression of SHF and hence may not necessarily add incremental value to AMSI determination using MIBG. Future studies with larger patient numbers are needed to definitively assess the incremental prognostic value of SPECT based LVMD estimation to AMSI in SHF patients.

\section{References}

[1] Ungerer, M., Bohm, M., Elce, J.S., et al. (1993) Altered Expression of Beta-Adrenergic Receptor Kinase and Beta 1-Adrenergic Receptors in the Failing Human Heart. Circulation, 87, 454-463. http://dx.doi.org/10.1161/01.CIR.87.2.454

[2] Caldwell, J.H., Link, J.M., Levy, W.C., et al. (2008) Evidence for Pre to Postsynaptic Mismatch of the Cardiac Sym- 
pathetic Nervous System in Ischemic Congestive Heart Failure. The Journal of Nuclear Medicine, 49, 234-241. http://dx.doi.org/10.2967/jnumed.107.044339

[3] Carrió, I., Cowie, M.R., Yamazaki, J., et al. (2010) Cardiac Sympathetic Imaging with mIBG in Heart Failure. JACC: Cardiovascular Imaging, 3, 92-100.

[4] Carrió, I. (2001) Cardiac Neurotransmission Imaging. The Journal of Nuclear Medicine, 42, 1062-1076.

[5] Jacobson, A.F., Senior, R., Cerqueira, M., et al. (2010) Myocardial Iodine-123 Meta-Iodobenzylguanidine Imaging and Cardiac Events in Heart Failure. Results of the Prospective ADMIRE-HF (AdreView Myocardial Imaging for Risk Evaluation in Heart Failure) study. Journal of the American College of Cardiology, 55, 2212-2221.

[6] Galt, J.R., Garcia, E.V. and Robbins, W.L. (1990) Effects of Myocardial Wall Thickness on SPECT Quantification. IEEE Transactions on Medical Imaging, 9, 144-150. http://dx.doi.org/10.1109/42.56338

[7] Somsen, G.A., Verberne, H.J., Burri, H., Ratib, O. and Righetti, A. (2006) Ventricular Mechanical Dyssynchrony and Resynchronization Therapy in Heart Failure: A New Indication for Fourier Analysis of Gated Bloodpool Radionuclide Ventriculography. Nuclear Medicine Communications, 27, 105-112.

http://dx.doi.org/10.1097/01.mnm.0000195671.90911.29

[8] Chen, J., Garcia, E.V., Bax, J.J., et al. (2011) SPECT Myocardial Perfusion Imaging for the Assessment of left Ventricular Mechanical Dyssynchrony. Journal of Nuclear Cardiology, 18, 685-694. http://dx.doi.org/10.1007/s12350-011-9392-x

[9] Trimble, M.A., Borges-Neto, S., Smallheiser, S., et al. (2011) Evaluation of Left Ventricular Mechanical Dyssynchrony as Determined by Phase Analysis of ECG-Gated SPECT Myocardial Perfusion Imaging in Patients with Left Ventricular Dysfunction and Conduction Disturbances. Journal of Nuclear Cardiology, 14, 298-307.

[10] Holly, T.A., Abbott, B.G., Al-Mallah, M., Calnon, D.A., Cohen, M.C., DiFilippo, F.P., Ficaro, E.P., Freeman, M.R., Hendel, R.C., Jain, D., Leonard, S.M., Nichols, K.J., Polk, D.M. and Soman, P. (2010) ASNC Imaging Guidelines for Nuclear Cardiology Procedures. Journal of Nuclear Cardiology, 17, 941-973.

[11] Abraham, W.T., Fisher, W.G., Smith, A.L., DeLurgio, D.B., Leon, A.R., Loh, E., et al. (2002) MIRACLE Study Group. Multicenter InSync Randomized Clinical Evaluation. Cardiac Resynchronization In Chronic Heart Failure. The New England Journal of Medicine, 346, 1845-1853. http://dx.doi.org/10.1056/NEJMoa013168

[12] Higgins, S.L., Hummel, J.D., Niazi, I.K., Giudici, M.C., Worley, S.J., Saxon, L.A., et al. (2003) Cardiac Resynchronization Therapy for the Treatment of Heart Failure in Patients with Intraventricular Conduction Delay and Malignant Ventricular Tachyarrhythmias. Journal of the American College of Cardiology, 42, 1454-1459. http://dx.doi.org/10.1016/S0735-1097(03)01042-8

[13] Young, J.B., Abraham, W.T., Smith, A.L., Leon, A.R., Lieberman, R., Wilkoff, B., et al. (2003) Multicenter InSync ICD Randomized Clinical Evaluation (MIRACLE ICD) Trial Investigators. Combined Cardiac Resynchronization and Implantable Cardioversion Defibrillation in Advanced Chronic Heart Failure: The MIRACLE ICD Trial. JAMA, 289, 2685-2694. http://dx.doi.org/10.1001/jama.289.20.2685

[14] McAlister, F., Ezekowitz, J., Wiebe, N., Rowe, B., Spooner, C., Crumley, E., et al. (2004) Cardiac Resynchronization Therapy for Congestive Heart Failure. Evid Rep Technol Assess, 1-8.

[15] Yonezawa, M., Nagao, M., Abe, K., Matsuo, Y., Baba, S., Kamitani, T., Isoda, T., Maruoka, Y., Jinnouchi, M., Yamasaki, Y., Abe, K., Higo, T., Yoshiura, T. and Honda, H. (2013) Relationship between Impaired Cardiac Sympathetic Activity and Spatial Dyssynchrony in Patients with Non-Ischemic Heart Failure: Assessment by MIBG Scintigraphy and Tagged MRI. Journal of Nuclear Cardiology, 20, 600-608. http://dx.doi.org/10.1007/s12350-013-9715-1

[16] Tanaka, H., Tatsumi, K., Fujiwara, S., Tsuji, T., Kaneko, A., Ryo, K., Fukuda, Y., Matsumoto, K., Shigeru, M., Yoshida, A., Kawai, H. and Hirata, K. (2012) Effect of Left Ventricular Dyssynchrony on Cardiac Sympathetic Activity in Heart Failure Patients With Wide QRS Duration. Circulation Journal, 76, 382-389.

[17] Yazaki, Y., et al. (2013) Risk Stratification of Sudden Cardiac Death by Evaluating Myocardial Sympathetic Nerve Activity Using Iodine-123 Metaiodobenzylguanidine Scintigraphy in Patients with Chronic Heart Failure and Dilated Cardiomyopathy. In: Milei, J. and Ambrosio, G., Eds., Cardiomyopathies, InTech. http://www.intechopen.com/books/cardiomyopathies/risk-stratification-of-sudden-cardiac-death-by-evaluating-myocar dial-sympathetic-nerve-activity-usin

[18] Chen, J., et al. (2011) SPECT Myocardial Perfusion Imaging for the Assessment of Left Ventricular Mechanical Dyssynchrony. Journal of Nuclear Cardiology, 18, 685-694. http://dx.doi.org/10.1007/s12350-011-9392-x 


\section{Submit or recommend next manuscript to SCIRP and we will provide best service for you:}

Accepting pre-submission inquiries through Email, Facebook, LinkedIn, Twitter, etc.

A wide selection of journals (inclusive of 9 subjects, more than 200 journals)

Providing 24-hour high-quality service

User-friendly online submission system

Fair and swift peer-review system

Efficient typesetting and proofreading procedure

Display of the result of downloads and visits, as well as the number of cited articles

Maximum dissemination of your research work

Submit your manuscript at: http://papersubmission.scirp.org/ 